\title{
EMPRENDIMIENTO como un pilar para la competitividad de las naciones
}

\section{ESUMEN}

En este artículo se hace referencia a la relación entre competitividad empresarial y emprendimiento, partiendo de algunas definiciones conceptuales y algunos estudios internacionales que han generado indicadores de medición de estos dos componentes. En este artículo se aborda el caso colombiano y se revisan indicadores de desempeño del Sistema Nacional de Creación e Incubación de Empresas (SNCIE).

\section{ialabras clave}

Emprendedor, productividad, competitividad, actividad emprendedora, empresas de base tecnológica, spin off.
Por:

Francisco Javier Matíz B. ${ }^{1}$

\section{Competitividad y creación de empresas}

El concepto de «competitividad» cobra cada vez más importancia y relevancia en los diferentes escenarios de nuestras sociedades, desde los círculos estatales hasta la academia, pasando por los medios de comunicación y obviamente por su principal gestor y responsable: el sector empresarial.

Uno de los principales autores sobre temas de gerencia y su relación con la competitividad y la productividad es el profesor Michael Porter para quien la productividad está determinada por el valor del producto generado por una unidad de trabajo o de capital. En su planteamiento, la productividad está en función de la calidad de los productos (de la que a su vez depende el precio) y de la eficiencia productiva.

\footnotetext{
Francisco Javier Matiz B.Coordinador del Programa Emprendedor. Docente investigador de la Universidad EAN. Profesional en Comercio Internacional. Especialista en psicología del consumidor y candidato a la maestría de Gestión de Organizaciones de las Universidades EAN y UQAC del Canadá. fmatiz.de@ean.edu.co
} 
Nos enfrentamos entonces a un concepto complejo que involucra no solo diversas acciones por parte de la empresa privada sino variables tanto políticas como económicas y macroeconómicas como el empleo y la demanda interior que se convierten en indicadores de la competitividad de nuestras naciones; un concepto que concierne a aspectos sociales como la educación y la formación para la gestión de las organizaciones.

Si buscamos niveles de competitividad basados en la productividad de los procesos y en la innovación de los productos, y no en esquemas de bajos precios y costos, está claro que las políticas de desarrollo deben estar orientadas fundamentalmente hacia aspectos claves como la formación, la innovación, la tecnología y la capacidad de emprendimiento de una sociedad.

Pero ¿puede la capacidad de emprendimiento de una sociedad afectar directamente sus niveles de productividad y competitividad? Este hecho puede explicarse por cuanto el proceso de creación de empresas además de ampliar el tejido empresarial con nuevas unidades productivas, en su gran mayoría (por no decir que en su totalidad) pequeñas y microempresas, aporta a indicadores como la disminución del desempleo y al aumento de la demanda interna. Las nuevas empresas se convierten en jalonadoras del aparato productivo, hecho claramente reconocido por el aporte de este tipo de empresas a la generación de empleo, al PIB y a la balanza comercial del país.

Nuevas empresas con enfoques de generación de valor, así como aquellas denominadas de base tecnológica, aportan significativamente al desarrollo de los sectores productivos mediante la incorporación de nuevas tecnologías, la implementación de nuevas

\section{A}

This article outlines the existent relationship between levels of economic development and competitiveness, entrepreneurship and the processes of creation of companies of a nation. It begins covering the topic and conceptualization of competitiveness, Then it stops and analize the quantification efforts of the start-ups processes of competitiveness and productivity of the countries, as the grade of development of the venturesome activity in the same ones, for this effect the author is centered in the reports of the World Economic Forum and the Global Entrepreneurship Monitor. Finally the Colombian case is analyzed, being supported in the reports of the National System of Creation and Incubation of Companies generated by the Presidency of the Republic.

\section{YEY WORDS}

Competitiveness, Productivity, Entrepreneurship, innovation, pymes, productive chains, entreprenurial activity, incubators of companies, internal agenda. 
propuestas de valor y la absorción de recurso humano altamente calificado. Algunas de las principales modalidades de generación de este tipo de compañías están asociadas a esquemas de spin off de medianas y grandes empresas, así como la articulación de las nuevas propuestas a cadenas y minicadenas productivas, como clusters, dinamizando de esta manera la gestión de sectores y regiones $y$, por ende, sirviendo de multiplicadores de la competitividad.

\section{Los indicadores}

Desde hace ya varios años diferentes instituciones se han preocupado por desarrollar indicadores que permitan medir tanto los niveles de competitividad como el desarrollo de la actividad emprendedora de las naciones. El seguimiento al desempeño frente a estas variables ha permitido establecer políticas y programas enfocados a favorecer el desempeño de los empresarios, así como esfuerzos enfocados a solucionar sus principales problemáticas.

Al referirnos a la competitividad empresarial debemos hacer referencia a los informes globales sobre competitividad, realizados anualmente por el Foro Económico Mundial y divulgados en el encuentro de este foro en la ciudad de Davos en Suiza.

El Foro Económico Mundial basa su informe de clasificación de las naciones con mayores niveles de competitividad en la percepción de más de 4.000 empresarios ejecutivos sobre los países donde operan sus compañías. Para la realización de este estudio el Foro tiene en cuenta ocho factores dentro de los cuales encontramos:
- Apertura

- Gobierno

- Finanzas

- Infraestructura

- Tecnología

- Administración

- Trabajo

- Instituciones

Cada uno de los países contemplado dentro del estudio es calificado en estos ocho aspectos $y$ finalmente se hace un promedio ponderado de estos factores para producir listado definitivo en el que se determina el índice general de competitividad.

Para el año 2005, de un muestreo de 117 países tenidos en cuenta en este estudio, los primeros lugares en función de competitividad y crecimiento económico fueron obtenidos por Finlandia, Estados Unidos y Suecia. Las últimas posiciones en este listado obedecen a Guyana, Kirguistán y Chad. En el caso latinoamericano, para el año 2005 el país con un mejor desempeño fue Chile seguido por México y Uruguay.

En el cuadro presentado en la siguiente página podemos observar los primeros 51 lugares en función de dos índices generales: crecimiento de la competitividad y competitividad actual.

De acuerdo con el Foro Económico Mundial (World Economic Forum) cada vez se tienen más en cuenta aspectos tales como el desarrollo de la tecnología de información en un país, lo cual se puede ver plasmado en temas como el uso del correo electrónico, la Internet y los teléfonos móviles. De acuerdo con los indicadores evaluados Escandinavia y Estados Unidos sobresalen como los líderes en este aspecto. 


\begin{tabular}{|c|c|c|}
\hline Crecimiento de la competitividad & Índices & Competitividad actual \\
\hline Finlandia & 1 & Finlandia \\
\hline Estados Unidos & 2 & Estados Unidos \\
\hline Canadá & 3 & Holanda \\
\hline Singapore & 4 & Alemania \\
\hline Australia & 5 & Suiza \\
\hline Noruega & 6 & Suecia \\
\hline Taiwán & 7 & Inglaterra \\
\hline Holanda & 8 & Dinamarca \\
\hline Suecia & 9 & Australia \\
\hline Nueva Zelandia & 10 & Singapur \\
\hline Irlanda & 11 & Canadá \\
\hline Inglaterra & 12 & Francia \\
\hline Hong Kong SAR & 13 & Austria \\
\hline Dinamarca & 14 & Bélgica \\
\hline Suiza & 15 & Japón \\
\hline Islandia & 16 & Islandia \\
\hline Alemania & 17 & Israel \\
\hline Austria & 18 & Hong Kong SAR \\
\hline Bélgica & 19 & Noruega \\
\hline Francia & 20 & Nueva Zelandia \\
\hline Japón & 21 & Taiwan \\
\hline España & 22 & Irlanda \\
\hline Korea & 23 & España \\
\hline Israel & 24 & Italia \\
\hline Portugal & 25 & Sudáfrica \\
\hline Italia & 26 & Hungría \\
\hline Chile & 27 & Estonia \\
\hline Hungría & 28 & Korea \\
\hline Estonia & 29 & Chile \\
\hline Malasia & 30 & Brasil \\
\hline Slovenia & 31 & Portugal \\
\hline Mauricio & 32 & Slovenia \\
\hline Tailandia & 33 & Turquía \\
\hline Sudáfrica & 34 & Trinidad y Tobago \\
\hline Costa Rica & 35 & República Checa \\
\hline Grecia & 36 & India \\
\hline República Checa & 37 & Malasia \\
\hline Trinidad y Tobago & 38 & Tailandia \\
\hline China & 39 & Slovakia \\
\hline República Eslovaca & 40 & Jamaica \\
\hline Polonia & 41 & Polonia \\
\hline México & 42 & Latvia \\
\hline Lituania & 43 & Grecia \\
\hline Brasil & 44 & Jordania \\
\hline Jordania & 45 & Egipto \\
\hline Uruguay & 46 & Uruguay \\
\hline Latvia & 47 & China \\
\hline Filipinas & 48 & Panamá \\
\hline Argentina & 49 & Lituania \\
\hline República Dominicana & 50 & Costa Rica \\
\hline Egipto & 51 & México \\
\hline
\end{tabular}


Estos esfuerzos de medición y seguimiento están concebidos para convertirse en «la herramienta más confiable e importante de que disponen las comunidades empresariales y los gobiernos para determinar sus políticas económicas y corporativas, a fin de entrar o quedarse en la liga más importante del mundo. En otras palabras, el Informe responde a las necesidades estratégicas de las compañías y los gobiernos y sirve como un instrumento útil para quienes toman decisiones políticas y de negocios» (World Economic Forum).

De igual forma y por iniciativa de las universidades Babson Collage y London Business School, desde el año 1997 se viene desarrollando el Global Entrepreneurship Monitor (GEM), un estudio internacional en el que especialistas en los temas referentes a emprendimiento y creación de empresas analizan la relación de estos procesos denominados en el estudio «actividad emprendedora» con el crecimiento y por ende desarrollo económico de los países objeto del estudio.

En sus inicio el GEM orientó sus esfuerzos de investigación a monitorear la actividad emprendedora en 10 países. A partir de esta fecha, año tras año se han vinculado más naciones hasta completar en el último informe del 2005 un total de 35 países analizados.

El informe anual del GEM se centra en trabajar sobre tres objetivos fundamentales:

- Medir en qué grado varía el nivel de actividad emprendedora entre los distintos países.

- Descubrir los factores que determinan el nivel de actividad emprendedora.

- Identificar políticas públicas para aumentar el nivel nacional de actividad emprendedora (GEM, 2005).

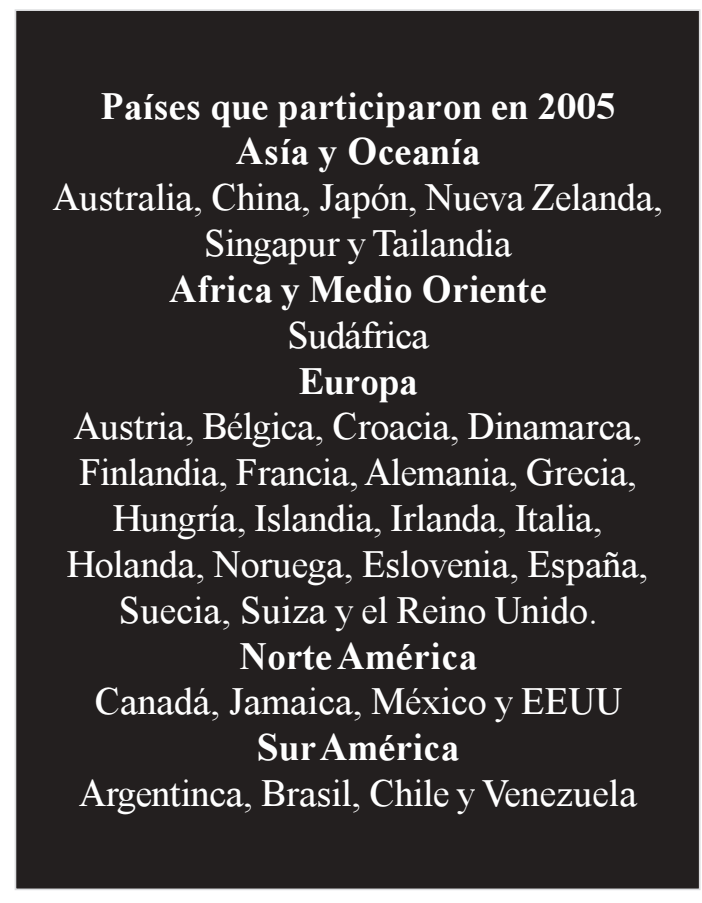

Para tal fin los investigadores del GEM utilizan como metodología la aplicación de instrumentos a emprendedores, así como entrevistas a profundidad con expertos relacionados con el tema. Según datos del informe del 2005, para este año el proceso contó con más de 100.000 encuestados y 1500 expertos entrevistados.

Dentro de los principales indicadores analizados por el GEM podemos encontrar aspectos asociados a las nuevas empresas tales como:

- Frecuencia y calidad de la actividad emprendedora.

- Cantidad de dueños de negocios establecidos.

- Supervivencia de las nuevas empresas. 
Aspectos relacionados con los emprendedores y sus características propias:

- Motivación de la creación de empresas (en este sentido el estudio determina dos grandes motivaciones: las empresas creadas a partir de oportunidades y aquellas surgidas por la necesidad).

- Características de edad, género y educación de los emprendedores.

Finalmente se hace seguimiento a lo que se podría determinar como la industria de apoyo a la creación de empresas en cada uno de los países analizados. En este sentido se hace un seguimiento y valoración de las políticas y programas de apoyo con que pueden contar los emprendedores.

A continuación podemos observar algunos de los resultados del estudio GEM para el año 2005: La aplicación de la investigación del GEM en un amplio número de países con características socioeconómicas diferentes permite analizar el comportamiento de las diferentes variables y su relación con el grado de desarrollo de estas economías.

A continuación encontramos algunas de las conclusiones realizadas a partir del estudio del 2005, relevantes a la hora de mirar la relación entre la creación de empresas y el desarrollo del emprendimiento con las condiciones de crecimiento y desempeño de las economías de cada país:

- Es evidente que existe una amplia variación entre países entre la frecuencia y la calidad de la actividad emprendedora. Esto se puede demostrar en que aquellos países de ingresos medios que hacen parte del estudio GEM generalmente muestran mayores porcentajes de emprendedores iniciando negocios, en relación con las cifras presentadas por los países con ingresos altos.

- Los países de ingresos medios están caracterizados principalmente por un mayor número de emprendimientos motivados por la necesidad, lo que es evidentemente diferente a los casos de los países de ingresos altos donde las principales motivaciones de los emprendimientos están en la oportunidad. Es importante también evidenciar el hecho de que los emprendimientos de oportunidad parecen tener una menor cantidad de fracasos en sus etapas tempranas.

- Por otra parte también se evidencia que los nuevos emprendedores tiene mayores posibilidades de sobrevivir en los países de ingresos altos, esto en gran medida gracias a la existencia de una industria de apoyo conformada por fondos de capital de riesgo, entidades de capacitación y asesoría, y centros de investigación muy ligados con el sector empresarial.

- Se evidencia como indicadores que las altas tasas de crecimiento de PBI per cápita en países de ingresos medios están reflejadas en las altas innovaciones y el crecimiento de potencial de la actividad emprendedora en este tipo de países.

- Frente a las características propias de los emprendedores, se encuentra que la mayoría de esta población se encuentra en individuos entre los 25 y 34 años, prevaleciendo los sujetos de género masculino, y con niveles de educación superior (generalmente universitaria). 


\section{Porcentaje de emprendedores en los países GEM 2005}

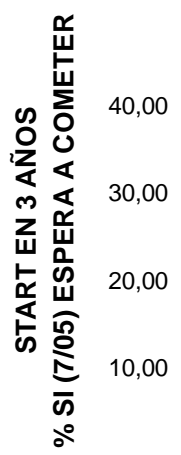

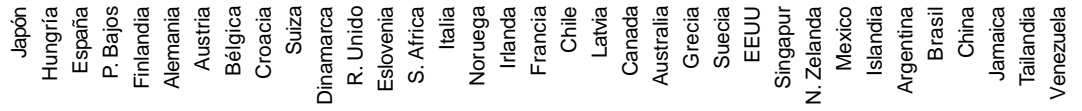

\section{Clasificación de los países GEM 2005 en función de su número de empresas early stage con potencial de rápido crecimiento en empleo}

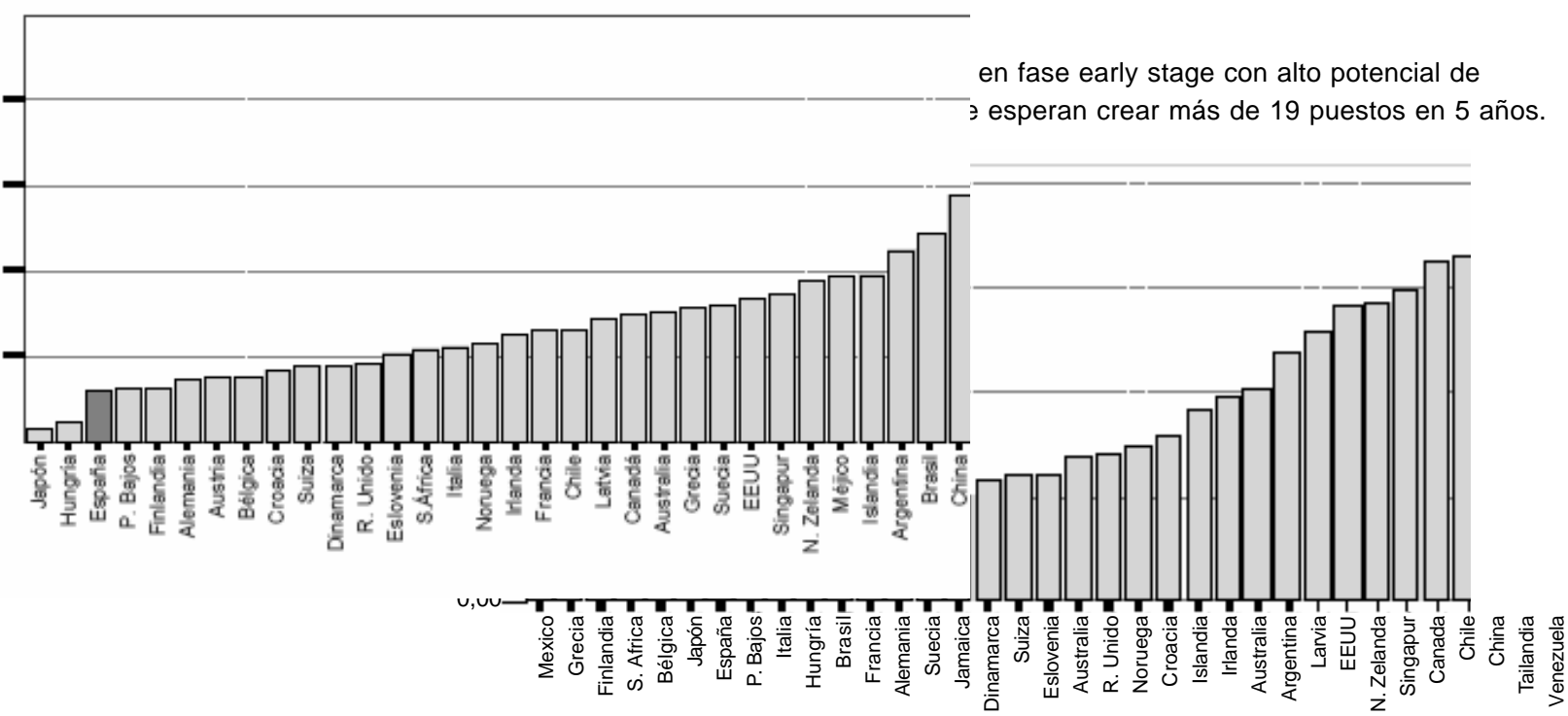

Países GEM 2005

La aplicación de la investigación del GEM en un amplio número de países con características socioeconómicas diferentes permite analizar el comportamiento de las diferentes variables y su relación con el grado de desarrollo de estas economías. 


\section{El caso colombiano}

Si bien Colombia no ha podido vincularse en ninguno de los estudios del GEM, y nacionalmente han sido pequeños y escasos los esfuerzos encaminados a realizar estudios de medición y seguimiento al impacto de la actividad de los emprendedores y de la creación de empresas en la economía nacional, en la actualidad a nivel nacional hay un claro compromiso con la causa emprendedora. Esta se evidencia no solo en los programas y políticas de apoyo a la creación de empresas y la generación de cultura emprendedora del gobierno nacional, sino en los esfuerzos igualmente valiosos adelantados por gobiernos locales (municipales y departamentales), así como por otro tipo de entidades como las organizaciones no gubernamentales (fundaciones y corporaciones) y principalmente por el sector académico donde en la actualidad la gran mayoría de instituciones tanto universitarias como de formación técnica y tecnológica han empezado a generar espacios para favorecer el desarrollo empresarial de sus usuarios.
Sin embargo datos sobre los que se puedan realizar algunos análisis referentes al impacto de este tipo de esfuerzos nos son muy abundantes. La Presidencia de la República, a través de su Sistema de Programación y Gestión (SIGOB), ha venido haciendo un seguimiento trimestral al denominado Sistema Nacional para la Creación e Incubación de Empresas (SNCIE), una iniciativa liderada por el Servicio Nacional de Aprendizaje (SENA) en la que se centran los resultados de la Red de Incubadoras de Empresas en las que esta entidad tiene participación como socio especial adherente.

Los principales indicadores medidos por la Presidencia de la República son el número de empresas creadas, los empleos generados y las ventas de las mismas. Vale la penar destacar que la mayoría de estas empresas son unidades empresariales de alto valor agregado. Muchas son empresas de base tecnológica o basadas en el conocimiento.

Fuente: SIGOB 
acompañamiento a emprendedores limitada, que la mayoría de los casos puede encontrarse en completa explotación.

Es por esta razón que el gobierno nacional desde al año 2005 viene apropiando recursos para fortalecer el SNCIE, favoreciendo la transferencia de modelos de incubación a las nuevas incubadoras y asegurando la expansión de la capacidad instalada de las instituciones ya consolidadas.

En cuanto a las características de las empresas creadas es importante destacar como, si bien son generadoras de empleo, su principal aporte está en la creación de nuevas plazas para empleados altamente calificados. Por su condición de empresas basadas en el conocimiento cuentan con equipos profesionales generadores de productos y servicios innovadores con un importante impacto. Esto puede evidenciarse en la correlación de los indicadores de empleos generados con las ventas facturadas por el conjunto de empresas. Sólo de esta manera es posible lograr que 890 nuevas empresas logren en un periodo de 4 años facturar más de 166 mil millones de pesos.

\section{Una reflexión}

Es evidente entonces la importancia tanto social como económica del «movimiento emprendedor» en cualquier país. Frente a este punto la Comisión Europea, a través de su Revista Innovación Europea, comenta: «Las nuevas empresas innovadoras de crecimiento rápido son esenciales para el desarrollo económico y para la creación de nuevos puestos de trabajo. Es evidente que sólo una pequeña parte de las nuevas empresas generará un número de puestos de trabajo significativo. Pero si los empresarios que hay detrás de ellas no tuvieran la ambición de crecer, su efecto sobre el empleo sería más atenuado"(Innovación Europea, Mayo, 2006). Se hacen necesarias entonces políticas claras frente al fomento al emprendimiento y la creación de empresas como una herramienta de Estado, no sólo para atacar problemas sociales como el desempleo, sino para fortalecer el aparato productivo en general.

Ejemplos como el Plan europeo a favor del espíritu empresarial (Plan Europeo, 2004) deben hacer reflexionar sobre este particular a los gobiernos de países de economías emergentes como Colombia. Un primer avance y de gran importancia sería el revisar los proyectos involucrados dentro de la Agenda Interna, coordinada por el Departamento Nacional de Planeación (DNP), para que tanto en las proyectos regionales como sectoriales sean tenidas en cuenta propuestas que permitan la generación de nuevas empresas.

Pero tal y como se ha evidenciado a lo largo de este artículo, éstas deben ser empresas innovadoras con altos componentes de valor agregado, cuya motivación sea el fruto de la oportunidad más que la necesidad, ya que en la actualidad encontramos dentro de las estadísticas de nuevas empresas un gran porcentaje de iniciativas surgidas frente a la necesidad, que en la mayoría de los casos terminan convirtiéndose en empresas de subsistencia.

Solo a través de la articulación de esfuerzos entre el sector productivo (empresarios y gremios), los gobiernos tanto locales como el nacional y, por su puesto, la academia, se podrán generar esfuerzos claros y representativos para articular iniciativas de creación de empresas a necesidades reales de mercado enmarcadas dentro de las cadenas, minicadenas productivas y clusters, $o$ en escenarios que permitan acercar la 
investigación aplicada a la industria mediante modelos de negocio como el spin off. Durante años el país se ha venido sensibilizando sobre la importancia del emprendimiento en los diferentes niveles. Es el momento de capitalizar este trabajo en una política de Estado que nos permita potencializar el espíritu emprendedor de nuestra sociedad.

\section{Bibliografía}

GEM (2005). Informe Ejecutivo GEM 2005 Centro de Entrepreneurship IAE - Escuela de Dirección y Negocios - Universidad Austral [Global Entrepreneurship Monitor] http:// www.gemconsortium.org/

document.asp?id=448

INNOVACIÓN EUROPEA (mayo, 2006) Comisión Europea. Dirección General de Empresa e Industria.

PLAN EUROPEO (2004). Plan Europeo a favor del espíritu empresarial - Comisión de las Comunidades Europeas - Bruselas 11.02.2004.
SIGOB [Sistema de Información y Seguimiento a las Metas del Gobierno]. Sistema Nacional de Incubación de Empresas. http://www.sigob.gov.co/pnd/ meta.aspx?m=100

WORLD ECONOMIC FORUM. Global Competitiveness Report 2006-2007. Accesible en http://www.weforum.org/en/initiatives/gcp/ Global\%20Competitiveness\%20Report/ index.htm [Foro Económico Mundial: Informe global en competitividad]. 
